\title{
A SEARCH FOR BRIEF OPTICAL FLASHES ASSOCIATED WITH THE SETI TARGET KIC 8462852
}

\author{
A. U. Abeysekara ${ }^{1}$, S. Archambault ${ }^{2}$, A. Archer ${ }^{3}$, W. Benbow ${ }^{4}$, R. Bird ${ }^{5}$, M. Buchovecky ${ }^{6}$, J. H. Buckley $^{3}$, K. Byrum $^{7}$, \\ J. V Cardenzana ${ }^{8}$, M. Cerruti ${ }^{4}$, X. Chen $^{9,10}$, J. L. Christiansen ${ }^{11}$, L. CiupiK ${ }^{12}$, W. Cui ${ }^{13}$, H. J. Dickinson ${ }^{8}$, J. D. Eisch ${ }^{8}$, \\ M. Errando ${ }^{14}$, A. FAlcone ${ }^{15}$, D. J. Fegan ${ }^{5}$, Q. Feng ${ }^{13}$, J. P. Finley ${ }^{13}$, H. Fleischhack ${ }^{10}$, P. Fortin $^{4}$, L. Fortson ${ }^{16}$, \\ A. Furniss ${ }^{17}$, G. H. Gillanders ${ }^{18}$, S. Griffin ${ }^{2}$, J. Grube ${ }^{12}$, G. GYuK ${ }^{12}$, M. Hütten ${ }^{10}$, N. HÅKANSSON ${ }^{9}$, D. HANnA ${ }^{2}$, \\ J. Holder ${ }^{19,20}$, T. B. Humensky ${ }^{21}$, C. A. Johnson ${ }^{22}$, P. KAARet ${ }^{23}$, P. KAR ${ }^{1}$, N. Kelley-Hoskins ${ }^{10}$, M. Kertzman ${ }^{24}$, D. KiedA ${ }^{1}$, \\ M. Krause ${ }^{10}$, F. Krennrich ${ }^{8}$, S. Kumar ${ }^{19}$, M. J. LANG ${ }^{18}$, T. T. Y. Lin ${ }^{2}$, G. Maier ${ }^{10}$, S. MCArThur ${ }^{13}$, A. McCANn ${ }^{2}$, \\ K. Meagher ${ }^{25}$, P. Moriarty ${ }^{18}$, R. Mukherjee ${ }^{14}$, D. Nieto ${ }^{21}$, S. O’Brien ${ }^{5}$, A. O’Faoláin de Bhróithe ${ }^{10}$, R. A. Ong ${ }^{6}$, \\ A. N. Otte ${ }^{25}$, N. Park ${ }^{26}$, J. S. Perkins ${ }^{27}$, A. Petrashyk ${ }^{21}$, M. Pohl ${ }^{9,10}$, A. Popkow ${ }^{6}$, E. PuescheL ${ }^{5}$ J. Quinn $^{5}$, K. Ragan ${ }^{2}$, \\ G. RatlifF ${ }^{12}$, P. T. Reynolds ${ }^{28}$, G. T. Richards ${ }^{25}$, E. RoAche ${ }^{4}$, M. SANTANDer ${ }^{14}$, G. H. Sembroski ${ }^{13}$, K. Shahinyan ${ }^{16}$, \\ D. Staszak ${ }^{2}$, I. Telezhinsky ${ }^{9,10}$, J. V. Tucci ${ }^{13}$, J. Tyler ${ }^{2}$, S. Vincent ${ }^{10}$, S. P. Wakely ${ }^{26}$, O. M. Weiner $^{21}$, \\ A. Weinstein ${ }^{8}$, D. A. Williams ${ }^{22}$, AND B. ZitZER ${ }^{7}$ \\ ${ }^{1}$ Department of Physics and Astronomy, University of Utah, Salt Lake City, UT 84112, USA \\ ${ }_{2}^{2}$ Physics Department, McGill University, Montreal, QC H3A 2T8, Canada \\ ${ }^{3}$ Department of Physics, Washington University, St. Louis, MO 63130, USA \\ ${ }^{4}$ Fred Lawrence Whipple Observatory, Harvard-Smithsonian Center for Astrophysics, Amado, AZ 85645, USA \\ ${ }^{5}$ School of Physics, University College Dublin, Belfield, Dublin 4, Ireland \\ ${ }^{6}$ Department of Physics and Astronomy, University of California, Los Angeles, CA 90095, USA \\ ${ }^{7}$ Argonne National Laboratory, 9700 S. Cass Avenue, Argonne, IL 60439, USA \\ ${ }^{8}$ Department of Physics and Astronomy, Iowa State University, Ames, IA 50011, USA; hughd@iastate.edu \\ ${ }_{9}$ Institute of Physics and Astronomy, University of Potsdam, D-14476 Potsdam-Golm, Germany \\ ${ }^{10}$ DESY, Platanenallee 6, D-15738 Zeuthen, Germany \\ ${ }_{11}^{11}$ Physics Department, California Polytechnic State University, San Luis Obispo, CA 94307, USA \\ 12 Astronomy Department, Adler Planetarium and Astronomy Museum, Chicago, IL 60605, USA \\ ${ }^{13}$ Department of Physics and Astronomy, Purdue University, West Lafayette, IN 47907, USA \\ ${ }^{14}$ Department of Physics and Astronomy, Barnard College, Columbia University, NY 10027, USA \\ ${ }^{15}$ Department of Astronomy and Astrophysics, Pennsylvania State University, 525 Davey Lab, University Park, PA 16802, USA \\ ${ }^{16}$ School of Physics and Astronomy, University of Minnesota, Minneapolis, MN 55455, USA \\ ${ }_{18}^{17}$ Department of Physics, California State University—East Bay, Hayward, CA 94542, USA \\ ${ }^{18}$ School of Physics, National University of Ireland Galway, University Road, Galway, Ireland \\ ${ }^{19}$ Department of Physics and Astronomy and the Bartol Research Institute, University of Delaware, Newark, DE 19716, USA; jholder@physics.udel.edu \\ ${ }^{20}$ Department of Physics and Space Science, Florida Institute of Technology, W. Melbourne, FL 32901, USA \\ ${ }^{21}$ Physics Department, Columbia University, New York, NY 10027, USA \\ ${ }^{22}$ Santa Cruz Institute for Particle Physics and Department of Physics, University of California, Santa Cruz, CA 95064, USA \\ ${ }^{23}$ Department of Physics and Astronomy, University of Iowa, Van Allen Hall, Iowa City, IA 52242, USA \\ ${ }^{24}$ Department of Physics and Astronomy, DePauw University, Greencastle, IN 46135-0037, USA \\ ${ }^{25}$ School of Physics and Center for Relativistic Astrophysics, Georgia Institute of Technology, 837 State Street NW, Atlanta, GA 30332-0430, USA \\ ${ }^{26}$ Enrico Fermi Institute, University of Chicago, Chicago, IL 60637, USA \\ ${ }^{27}$ NASA Goddard Space Flight Center, Code 661, Greenbelt, MD 20771, USA \\ ${ }^{28}$ Department of Physical Sciences, Cork Institute of Technology, Bishopstown, Cork, Ireland \\ Received 2016 January 9; accepted 2016 February 2; published 2016 February 18
}

\begin{abstract}
The F-type star KIC 8462852 has recently been identified as an exceptional target for search for extraterrestrial intelligence (SETI) observations. We describe an analysis methodology for optical SETI, which we have used to analyze nine hours of serendipitous archival observations of KIC 8462852 made with the VERITAS gamma-ray observatory between 2009 and 2015. No evidence of pulsed optical beacons, above a pulse intensity at the Earth of approximately 1 photon $\mathrm{m}^{-2}$, is found. We also discuss the potential use of imaging atmospheric Cherenkov telescope arrays in searching for extremely short duration optical transients in general.
\end{abstract}

Key words: astrobiology - extraterrestrial intelligence - methods: observational - stars: individual (KIC 8462852) - techniques: photometric

\section{INTRODUCTION}

Over the course of its four-year primary mission, NASA's Kepler spacecraft provided photometric measurements of over 150,000 stars, typically sampled every 30 minutes. A number of the resulting high-precision light curves exhibit unusual variability patterns, which can generally be explained as the result of analysis artifacts or by known astrophysical mechanisms. Boyajian et al. (2015) recently provided an in-depth study of KIC 8462852 (TYC 3162-665-1), a star whose light curve was flagged as unusual by members of the Zooniverse citizen science Planet Hunters project (Fischer et al. 2012). They identify the star as a main-sequence F3 V/IV star and describe unique, aperiodic dips in the stellar flux of up to $20 \%$, lasting between 5 and 80 days. Archival photographic plates also show unprecedented century-long dimming, at an average rate of $0.165 \pm 0.013 \mathrm{mag}$ per century (Schaefer 2016). One possible explanation is that the observations may be explained by the passage of a family of exocomet fragments resulting from a single break-up event (Bodman \& Quillen 2015; Boyajian et al. 2015). Wright et al. (2016) offer an alternative "extraordinary 

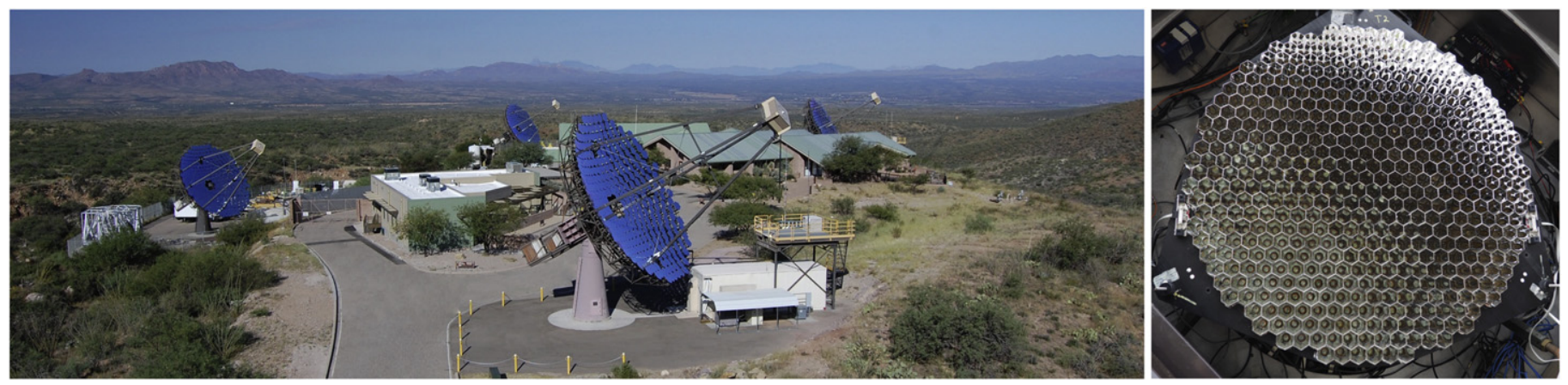

Figure 1. The VERITAS array in Arizona (left) and the PMT camera of a single telescope (right). The camera covers a total field of view of diameter 3.5 . The hexagonal reflective light cones reduce the dead space between the circular PMTs.

hypothesis" that the light curve is consistent with the existence of a collection of planet-sized structures, or swarms of many smaller objects, placed in orbit by an extraterrestrial civilization. They describe KIC 8462852 as an outstanding target for search for extraterrestrial intelligence (SETI) observations. Radio frequency observations between 1 and $10 \mathrm{GHz}$ using the Allen Telescope Array from 2015 October 15 to 30, for approximately $12 \mathrm{hr}$ each day, did not find any evidence of a signal (Harp et al. 2015).

Astronomical SETI observations are most commonly conducted at radio frequencies, close to the $21 \mathrm{~cm}$ hydrogen line. However, Schwartz \& Townes (1961) noted over half a century ago that optical light sources are also effective beacons, detectable over interstellar distances. One promising method is to search for intense pulses of optical or near-infrared photons from candidate star systems: Howard et al. (2004) have calculated that, using current technology $(10 \mathrm{~m}$ reflectors as the transmitting and receiving apertures and a 3.7 MJ pulsed laser source), a 3 ns optical pulse could be produced that would be easily detectable at a distance of 1000 ly, outshining starlight from the host system by a factor of $10^{4}$. A number of dedicated searches for such signals have been conducted or are under development (e.g., Howard et al. 2004, 2007; Stone et al. 2005; Wright et al. 2014). Schuetz et al. (2015) performed a search for periodic optical pulses from KIC 8462852 between 2015 October 29 and November 9, using the $0.5 \mathrm{~m}$ telescope of the Boquete Optical SETI Observatory, with null results. Alternative approaches to optical SETI include searching for the spectral signatures of laser emission either in the form of extremely narrow emission lines (Tellis \& Marcy 2015) or as periodic signatures in the spectra (Borra 2012).

The detection of nanosecond optical pulses from the night sky requires large-aperture mirrors instrumented with fast photon detectors. Ground-based gamma-ray telescopes have identical requirements and can be used to search for SETI-like signals (Covault 2001; Eichler \& Beskin 2001; Armada et al. 2005; Holder et al. 2005; Hanna et al. 2009). The requirement for coincident signals between multiple independent telescopes, combined with the ability to form a crude image of the light flash, allows imaging atmospheric Cherenkov telescopes (IACTs) to perform such searches parasitically during regular observations, with effectively no background. We report here on the results of observations of KIC 8462852, recorded serendipitously by the VERITAS gamma-ray observatory.

\section{VERITAS INSTRUMENT AND OBSERVATIONS}

VERITAS (Weekes et al. 2002) is an array of four IACTs located at the Fred Lawrence Whipple Observatory in southern Arizona (Figure 1). The telescope optics follow a DaviesCotton design (Davies \& Cotton 1957), with a $12 \mathrm{~m}$ aperture reflector and a $12 \mathrm{~m}$ focal length. The reflector comprises 345 hexagonal mirror facets, giving a total mirror area of $110 \mathrm{~m}^{2}$. The telescopes are mounted on steerable alt-azimuth positioners and are arranged in an approximate diamond formation with sides of roughly $100 \mathrm{~m}$ length. At the focus of each telescope is a close-packed array of 499 photomultiplier tubes (PMTs; Figure 1). The PMT angular spacing is 0.15 , giving a roughly circular field of view for each telescope of diameter 3.5. Dead space between the PMTs is removed by the addition of reflecting hybrid-Winston cones to the PMT front faces, with a hexagonal shape at the entrance and a circular exit window (Nagai et al. 2008). The photodetectors (initially Photonis XP2970 PMTs, upgraded to Hamamatsu R10560 Super Bialkali PMTs in 2012) are sensitive throughout the visible wavelength range, with a peak detection efficiency around $400 \mathrm{~nm}$.

Ground-based gamma-ray astronomy is an indirect technique, which works by forming an image of cascades of relativistic particles in the Earth's atmosphere ("air showers") using the few-nanosecond pulse of optical Cherenkov light that they generate. The telescopes therefore do not integrate light continuously; rather, the PMT signals are split, with one copy passed to a fast multi-level trigger system. A telescope trigger is generated when the signals in at least three adjacent PMTs cross a discriminator threshold within approximately $5 \mathrm{~ns}$. An array trigger is generated when at least two of the four telescopes trigger within a coincidence window of $50 \mathrm{~ns}$. On receipt of a successful array trigger, all of the PMT signals are read out using flash analog-digital converters (FADCs) that sample the signal every $2 \mathrm{~ns}$. The trigger rate is typically a few hundred $\mathrm{Hz}$, almost all of which is due to cosmic-rayinitiated particle cascades. The VERITAS telescope design is described in more detail in Holder et al. (2006), and the IACT technique is summarized in Holder (2015).

VERITAS observations are made at night, under clear skies. The telescopes are operated in concert and track gamma-ray source candidates as they move in azimuth and elevation. The standard observing mode consists of an exposure of 15-30 minutes, with the target offset from the center of the field of view by 0.5 , sequentially toward the north, south, east, and west. The telescope positioning is accurate to a few arcminutes, and offline corrections using CCD star trackers determine 
Table 1

VERITAS Observations of KIC 8462852

\begin{tabular}{|c|c|c|c|c|c|}
\hline MJD & $\begin{array}{c}\text { Start } \\
\text { (UTC) }\end{array}$ & $\begin{array}{c}\text { End } \\
\text { (UTC) }\end{array}$ & $\begin{array}{c}\text { Offset } \\
\text { Direction }\end{array}$ & $\begin{array}{l}\text { Elevation } \\
\text { Midpoint }\end{array}$ & Comments \\
\hline 55143 & $1: 40$ & 2:00 & $\mathrm{N}$ & $67^{\circ} .4$ & $\ldots$ \\
\hline 55143 & $2: 02$ & $2: 22$ & $\mathrm{~S}$ & 64.2 & $\ldots$ \\
\hline 55144 & $1: 58$ & $2: 18$ & $\mathrm{~W}$ & 63.6 & $\ldots$ \\
\hline 55145 & $1: 52$ & $2: 12$ & $\mathrm{~N}$ & 64.1 & $\ldots$ \\
\hline 55146 & $1: 37$ & $1: 57$ & $\mathrm{~S}$ & 66.5 & $\ldots$ \\
\hline 55146 & $1: 58$ & $2: 18$ & $\mathrm{E}$ & 63.2 & $\cdots$ \\
\hline 55151 & $1: 35$ & $1: 55$ & $\mathrm{~W}$ & 62.8 & $\ldots$ \\
\hline 55151 & $1: 56$ & $2: 16$ & $\mathrm{~N}$ & 59.5 & $\ldots$ \\
\hline 55152 & $1: 45$ & $2: 02$ & $\mathrm{~S}$ & 61.3 & $\ldots$ \\
\hline 55152 & $2: 05$ & $2: 26$ & $\mathrm{E}$ & 57.7 & $\ldots$ \\
\hline 55326 & $10: 02$ & $10: 22$ & $\mathrm{E}$ & $63^{\circ} 0$ & 3 telescopes \\
\hline 55326 & $10: 23$ & $10: 43$ & $\mathrm{~W}$ & $67^{\circ} .4$ & 3 telescopes \\
\hline 55326 & $10: 44$ & $11: 12$ & $\mathrm{~N}$ & 70.6 & 3 telescopes \\
\hline 55356 & $10: 06$ & $10: 26$ & $\mathrm{~S}$ & 78.1 & $\ldots$ \\
\hline 55357 & $10: 33$ & $10: 53$ & $\mathrm{~S}$ & 76.4 & $\ldots$ \\
\hline 55381 & $6: 36$ & $6: 56$ & $\mathrm{~N}$ & $65^{\circ} .1$ & $\ldots$ \\
\hline 55381 & $6: 57$ & $7: 17$ & $\mathrm{~S}$ & $68^{\circ} .9$ & $\ldots$ \\
\hline 56091 & $9: 15$ & $9: 35$ & $\mathrm{~N}$ & $75^{\circ} 8$ & $\ldots$ \\
\hline 56091 & $9: 36$ & $9: 56$ & $\mathrm{~S}$ & 77.9 & $\ldots$ \\
\hline 56203 & $4: 45$ & $5: 05$ & $\mathrm{~S}$ & 60.2 & $\cdots$ \\
\hline 56943 & 2:08 & $2: 23$ & $\mathrm{~N}$ & 76.8 & $\cdots$ \\
\hline 56953 & $1: 59$ & $2: 14$ & $\mathrm{~S}$ & $75^{\circ} 0$ & $\ldots$ \\
\hline 56974 & $1: 44$ & $1: 59$ & E & $64: 8$ & $\ldots$ \\
\hline 56981 & $1: 38$ & $1: 53$ & W & 60.1 & $\ldots$ \\
\hline 57162 & $9: 55$ & $10: 10$ & $\mathrm{~N}$ & $68^{\circ} .0$ & $\ldots$ \\
\hline 57189 & $8: 49$ & 9:04 & $\mathrm{S}$ & 74.5 & $\ldots$ \\
\hline 57283 & $4: 21$ & $4: 36$ & $\mathrm{~N}$ & 73.8 & $\ldots$ \\
\hline 57297 & $2: 37$ & $2: 52$ & $\mathrm{~N}$ & $77^{\circ} .2$ & $\ldots$ \\
\hline
\end{tabular}

Note. The horizontal break indicates when the telescope PMTs were upgraded. All four telescopes were operating, unless otherwise stated.

individual telescope pointing directions to better than 20 arcsec.

Given the wide field of view of the instrument, it is common for interesting targets to be observed serendipitously. KIC 8462852 (R.A. $20^{\mathrm{h}} 06^{\mathrm{m}} 15$ s.46, decl. +44 $27^{\prime} 24^{\prime \prime}$ 6) lies 1.07 from 0FGL J2001.0+4352 (R.A. $20^{\mathrm{h}} 01^{\mathrm{m}} 12^{\mathrm{s}} .87$, decl. $+43^{\circ} 52^{\prime} 52^{\prime \prime}$ ) ), a gamma-ray source associated with an active galactic nucleus (Aleksić et al. 2014). The VERITAS archive contains observations of this region taken between 2009 and 2015, with KIC 8462852 offset from the center of the field of view by $0.87,1.37,0.67$, and 1.48 for the $\mathrm{N}, \mathrm{S}, \mathrm{E}$, and $\mathrm{W}$ offsets, respectively. Table 1 lists the VERITAS observations of KIC 8462852. The total exposure, after selecting only goodweather runs with no major hardware problems, is 526 minutes.

\section{ANALYSIS AND RESULTS}

Data calibration and image pre-processing follow the same procedures used for gamma-ray observations with VERITAS (Holder et al. 2006). The PMT responses are first flat-fielded using a pulsed LED source (Hanna et al. 2010). For each event, PMTs containing significant signal are identified, and the resulting images are parameterized with an ellipse. The image properties: its rms length and width, and its orientation and intensity (Hillas 1985), are then used to classify the events and, in the case of gamma-ray and cosmic-ray images, to reconstruct the properties of the cascade progenitor.
In this analysis, we are searching for evidence of pulsed emission from a distant optical beacon. The resulting images would have a number of characteristics that make them simple to identify. Specifically:

1. They appear in the same place in all four telescope cameras.

2. They have the same intensity in each telescope.

3. They are point-like, that is, they have the same morphology as the telescope optical point-spread function (PSF).

These criteria alone are sufficient to remove essentially all of the background cosmic-ray events from the analysis. Cosmicray air showers produce the majority of their Cherenkov light below an altitude of $20 \mathrm{~km}$, and so the images recorded in detectors separated by $100 \mathrm{~m}$ show significant parallactic displacement $(>0.3)$. Image centroid locations can be measured with a precision typically an order of magnitude better than this. Cosmic-ray showers develop and grow longitudinally in the atmosphere, producing Cherenkov light over a typical length of several kilometers and resulting in an image with a large angular extent (Figure 2). Furthermore, an optical beacon originating from KIC 8462852 would produce images with centroid locations consistent with the location of the star in the telescope cameras. The most convincing signal would be given by multiple occurrences of such images at different times, each matching the star's position.

One concern is whether such images would successfully trigger the telescope readout, which requires at least three adjacent PMTs in each telescope camera to have signals exceeding their discriminator thresholds. The optical PSF at the center of the field of view has a typical full width at half maximum of 0.06 (or 0.09 at the $68 \%$ containment radius), with almost all of the light in the image of a point source contained within the diameter of a single PMT $\left(0^{\circ} .15\right)$. However, as a natural result of the alt-azimuth design of the telescopes, a celestial source moves across the field of view and spends a reasonable fraction of time at the interface between two or three PMT pixels. Furthermore, the optical PSF degrades significantly off the optical axis, blurring and distorting the image of a point source more widely near the outer edge of the camera (by approximately $50 \%$ at $1^{\circ}$ off-axis). This increases the probability that a point-like image will generate a trigger.

The temporal characteristics of the light pulse also play an important role in determining the trigger efficiency. The PMT signal path is AC-coupled in the telescope camera prior to preamplification, with a lower cutoff frequency of approximately $100 \mathrm{kHz}$. A steady, or slowly varying, signal will not generate a trigger. There is a caveat to this, in that a steady optical source such as a bright star in the field of view will generate highfrequency Poisson noise fluctuations in the PMT signals, increasing the probability of accidentally crossing the discriminator thresholds. KIC 8462852 itself has a V-band magnitude of 12.01 (Høg et al. 2000), which is too faint to produce any measurable increase in the noise fluctuations of the PMT signal. An optical pulse with a duration much longer than the AC-coupling time constant, but with a risetime significantly less than $10 \mu \mathrm{s}$, will also trigger the cameras.

Based on these criteria, we have processed all of the data to search for optical pulses associated with KIC 8462852. For every recorded event we have applied selection cuts based on 

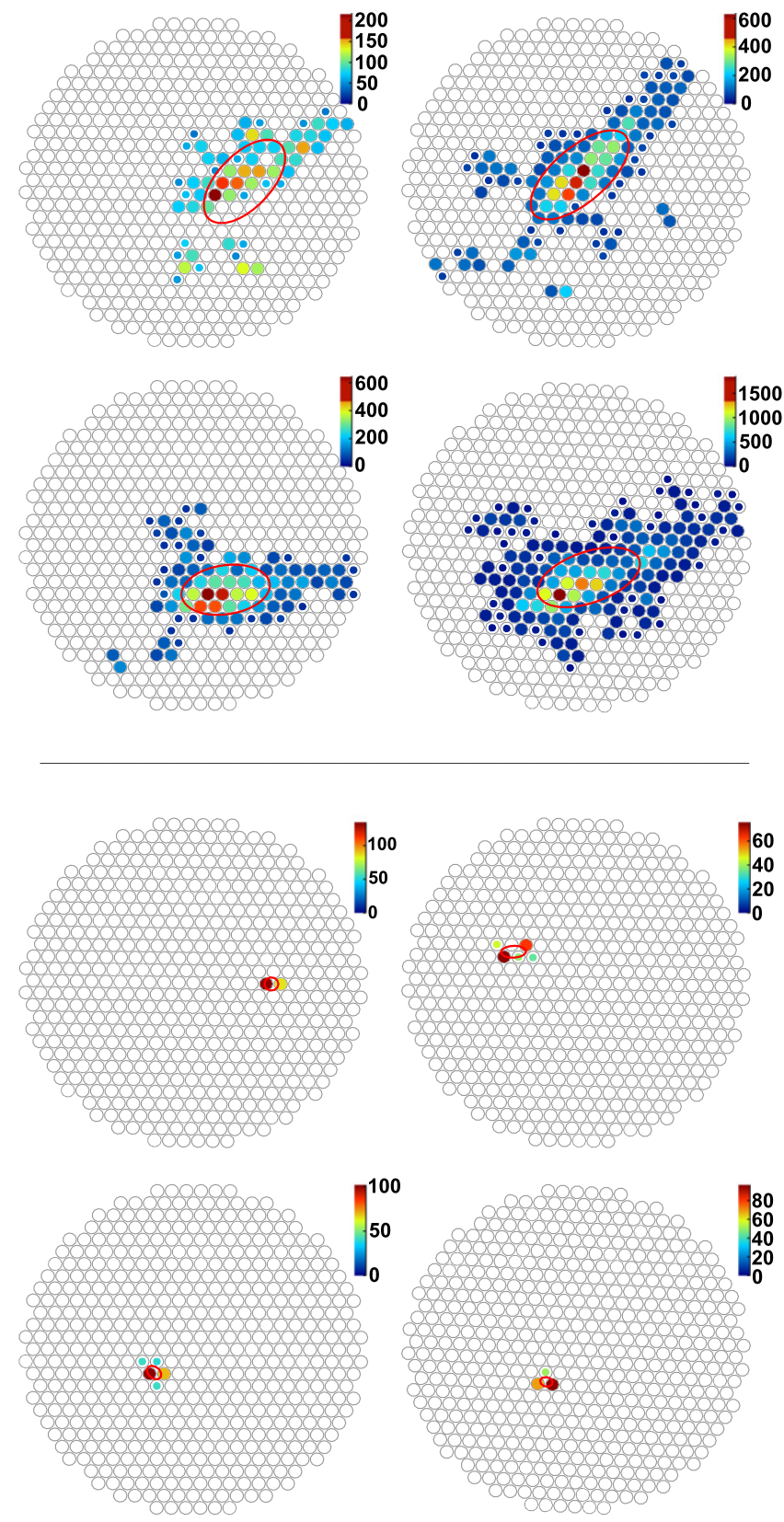

Figure 2. Cosmic-ray events in the four VERITAS multi-pixel PMT cameras. The diameter of the field of view is 3.5, and the color scale indicates the intensity of Cherenkov light in each PMT in FADC digital counts (where 5.3 digital counts corresponds to one photo-electron). The top panel shows an event initiated by a high-energy cosmic ray, with large angular extent. The bottom panel shows a fainter, lower-energy cosmic-ray event, which is still easily distinguished from a distant point source due to the large parallactic displacement between the image locations in each camera.

the recorded images. We retained events in which all images had ellipse lengths and widths less than $0^{\circ} .1125$, equivalent to the length of an image with three aligned PMTs each containing exactly the same signal intensity. We also require that at least three telescopes record an image, and that no two image centroid locations within the same event are separated by more than 0.15 , equivalent to the spacing between adjacent PMTs.

Inspection of the surviving events reveals that the majority resemble faint cosmic-ray events, or noise fluctuations, typically with low-intensity images. A four-telescope image multiplicity requirement, or a minimum image intensity cut, efficiently removes these. These additional criteria, together with a requirement for uniform intensity across all images, could be be applied in future analyses requiring stricter background rejection, such as searches without a predefined source candidate location.

Interestingly, some point-like optical pulse candidates are observed. In particular, during a 15 minute exposure on 2015 September 18 (MJD 57283), two different classes of pointsource optical pulses are detected. One of these consists of around 300 consecutive events, which move in a straight line across the field of view, crossing $3^{\circ} .4$ in $0.11 \mathrm{~s}$. The images are not co-located in the field of view, displaying a maximum parallactic displacement of 0.1 , observed between two telescopes separated by $150 \mathrm{~m}$. This implies an altitude of emission of $86 \mathrm{~km}$ and a velocity of roughly $50 \mathrm{~km} \mathrm{~s}^{-1}$, consistent with the typical properties of the light from a meteoroid passing through the Earth's thermosphere. We note that the precursor to VERITAS, the original Whipple $10 \mathrm{~m}$ gamma-ray telescope, was used to provide the first optical observations of meteors down to a limiting photographic magnitude of +12 in the 1970s (Cook et al. 1980). Another class of optical pulses, an example of which is seen in the same exposure, are more difficult to explain. These consist of eight events, recorded with the telescopes pointing toward $323^{\circ} .7$ azimuth and $73^{\circ} .3$ elevation. The events again move in a straight line across the field of view, crossing $2^{\circ} .2$ in $28.9 \mathrm{~s}$, from 04:33:01 to 04:33:30 UT (Figure 3). There is no measurable parallactic displacement in this case, implying an altitude of at least $200 \mathrm{~km}$. The pulse duration is longer than the FADC readout window length of $32 \mathrm{~ns}$. We consider that the most likely explanation is that these events are due to light reflected from a satellite; the measured angular velocity $\left(4.6 \mathrm{~s}^{-1}\right)$ is consistent with an object in orbit at an altitude of a few thousand kilometers. Events such as these, which form tracks, are easily distinguished from a true celestial source, simply by their motion across the field of view. They also serve as a useful proof of principle, demonstrating that the array does trigger on point-like optical sources at large distances, and highlighting the advantages of a widely separated telescope array with imaging capabilities and a large field of view in avoiding misclassification.

After applying the selection cuts, and removing any event sequences that form tracks in the cameras, only 28 of the initial $7,036,970$ events remain -1 in every 251,320 events. None of these have images consistent with the location of KIC 8462852. We conclude that there is no evidence in the VERITAS observations for optical pulses originating from this system.

\section{DISCUSSION}

Hanna et al. (2009) have already outlined the difficulty in forming meaningful constraints from optical SETI observations. In particular, aside from the very obvious reasons for the lack of a detection (i.e., there is very likely no extraterrestrial civilization at KIC 8462852 directing laser pulses toward us), the potentially transient nature of any signal implies that the same observations could be performed at a different time, with a different result. Nevertheless, with nine hours of observations distributed over six years, the VERITAS archive represents a unique data set. 

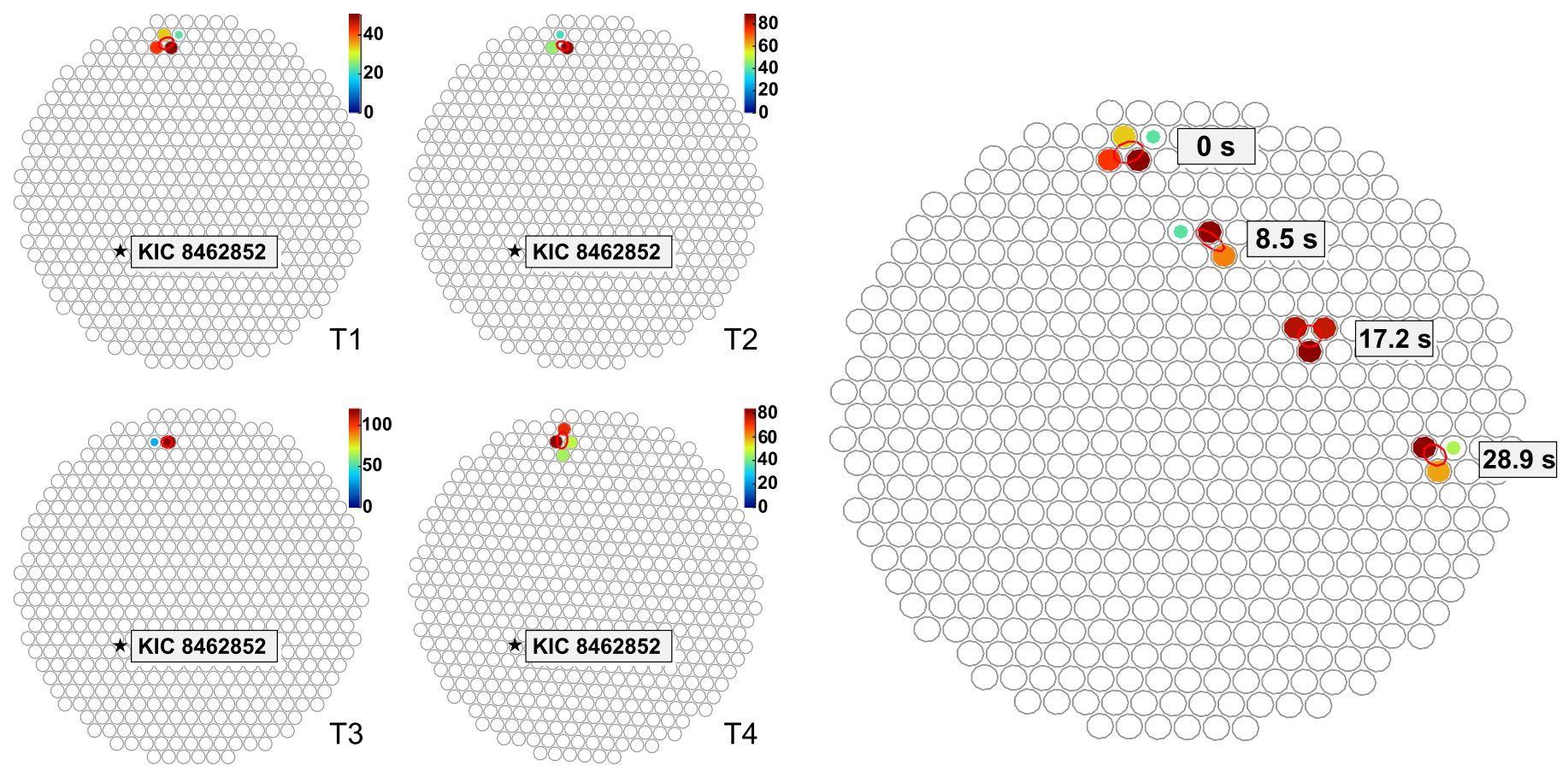

$8.9 \mathrm{~s}$

Figure 3. Point-like events generated by an object moving across the field of view of VERITAS over the course of $28.9 \mathrm{~s}$ on MJD 57283. Left: a single event viewed by all four telescopes. Right: a subset of the eight recorded events illustrating the motion of the image across the camera of a single telescope.

An exact estimate of the sensitivity of the search is also difficult since the minimum detectable optical pulse intensity depends strongly upon the emission wavelength, the duration and temporal profile of the pulse, and the exact source location within the field of view when the pulse occurs-all of which are unknown. Taking representative values (20\% photon detection efficiency, $85 \%$ mirror reflectivity, and a conservative minimum image intensity of 100 digital counts, corresponding to 18.8 photo-electrons) gives a minimum optical pulse intensity of $0.94 \mathrm{ph} \mathrm{m}^{-2}$ arriving at the telescope within the $12 \mathrm{~ns}$ pulse integration window used for this analysis. The required energy of the transmitted pulse, estimated using the method of Howard et al. (2004), is $0.3 \mathrm{MJ}$, or roughly equivalent to a $B$-band $6.4 \mathrm{mag}$ star, but with just a few nanoseconds duration. This compares favorably with earlier searches with optical telescopes, which have sensitivities of typically $60-100 \mathrm{ph} \mathrm{m}^{-2}$, and with observations by the STACEE heliostat array telescope, which provided a sensitivity of $10 \mathrm{ph} \mathrm{m}^{-2}$ (Hanna et al. 2009). The recent optical SETI observations of KIC 8462852 by Schuetz et al. (2015) report a sensitivity to periodic signals of $67 \mathrm{ph} \mathrm{m}^{-2}$.

We can also estimate the wavelength dependence of the sensitivity, taking into account the effects of interstellar absorption, transmission through the Earth's atmosphere, the reflectivity of the telescope mirrors, and the quantum efficiency of the photodetectors. Boyajian et al. (2015) calculate a distance to KIC 8462852 of 454 pc, based upon the interstellar reddening of the spectral energy distribution $(E(B-V)=0.11 \pm 0.03 \mathrm{mag})$, which corresponds to a $V$ band extinction of $A_{V}=0.341 \mathrm{mag}$, or a transmission efficiency of $73 \%$. We normalize wavelength-dependent extinction values (Schlafly \& Finkbeiner 2011) to this measurement, as shown in Figure 4. The overall wavelength dependence of the sensitivity is given by the "Total" curve.

Perhaps the most important conclusion of this work is that modern IACT arrays are effective tools to search for faint optical

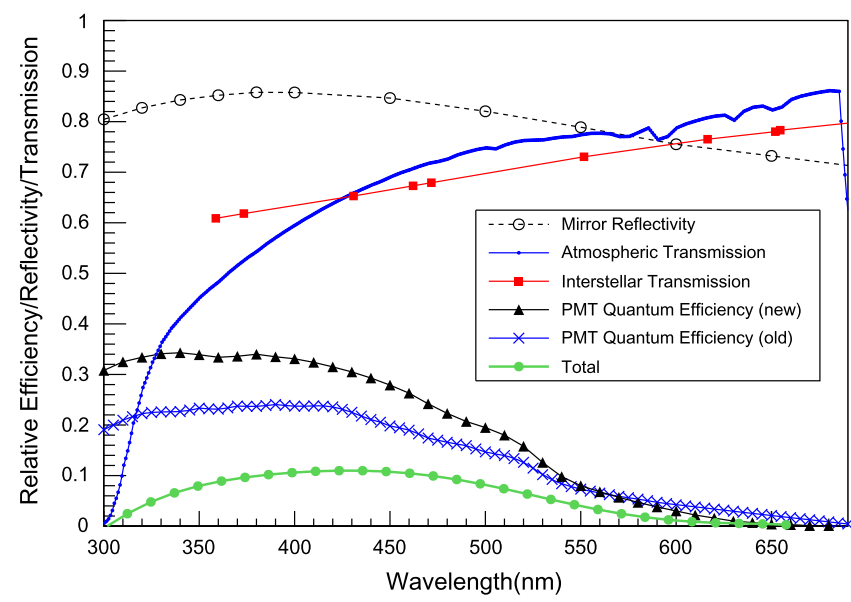

Figure 4. The wavelength-dependent efficiency for detection of an optical beacon by VERITAS, illustrating the effects of interstellar reddening, the Earth's atmosphere (generated using MODTRAN; Berk et al. 2006), the mirror reflectivity (VERITAS measurement), and the PMT quantum efficiency both before and after the PMT upgrade in 2012 (VERITAS measurement). The "Total" curve multiplies these effects together (assuming the post-2012 quantum efficiency), giving a peak efficiency for detection at $420 \mathrm{~nm}$.

transients, with durations as brief as a few nanoseconds. The observations are complementary to, and have no impact on, the scientific program in the gamma-ray domain. Any artificial backgrounds can be easily removed, using the simple analysis procedures outlined here. The search for short-duration optical transients was highlighted as a fruitful area of research almost half a century ago (e.g., Bondi 1970 and Schaefer et al. 1984) and has developed into an important field of astronomy over the past decade, with many different scientific goals (e.g., Schmidt 2012 lists gravitational microlensing, asteroid detection, stellar variability, extragalactic transients, and cosmology using supernovae). Dedicated robotic arrays of astrographs, or reflecting telescopes with Schmidt optics, monitor the skies nightly to 
explore the time domain (e.g., the Catalina Real-Time Transient Survey, the Hungarian Automated Telescope Network, the Palomar Transient Factory, etc.) while, in the near future, the Large Synoptic Survey Telescope will map the entire visible sky every few nights with pairs of $15 \mathrm{~s}$ exposures, separated by 15-60 minutes and reaching a $5 \sigma$ sensitivity limit of $24.5 \mathrm{mag}$ (Ivezic et al. 2008). Extending this search to nanosecond timescales opens up a new area of parameter space. While shortduration gamma-ray bursts have now been studied for decades, the surprising recent discovery of millisecond-timescale fast radio bursts (Lorimer et al. 2007) and nanosecond radio pulses from the Crab pulsar (Hankins et al. 2003) demonstrates that such rapid phenomena may exist elsewhere in the electromagnetic spectrum. Further motivation is provided by Borra (2013), who has discovered evidence for rapid periodic modulations in the optical spectra of galaxies, while Lacki (2014) has noted the potential of IACTs for rapid photometry of bright sources, particularly in the context of observing stellar occultations by small objects in the outer solar system.

VERITAS has been operating since 2007 and records over $1000 \mathrm{hr}$ of observations per year. Together with the H.E.S.S. array in Namibia and the MAGIC system in La Palma, approximately $30,000 \mathrm{hr}$ of archived IACT data exist and a substantial fraction of the entire sky has been observed. A search of these archives for optical transients seems worthwhile. Beyond the current generation of instruments lies the planned Cherenkov Telescope Array (CTA), which will consist of northern and southern hemisphere telescope arrays on a much larger scale, totaling over 100 telescopes (Acharya et al. 2013). CTA will provide an enormous increase in mirror area and telescope multiplicity, with the potential to greatly enhance searches for optical transients. This additional application of the facility can inform design decisions regarding the telescope optics and trigger systems.

This research is supported by grants from the U.S. Department of Energy Office of Science, the U.S. National Science Foundation, and the Smithsonian Institution, and by NSERC in Canada. We acknowledge the excellent work of the technical support staff at the Fred Lawrence Whipple Observatory and at the collaborating institutions in the construction and operation of the instrument. The VERITAS Collaboration is grateful to Trevor Weekes for his seminal contributions and leadership in the field of VHE gamma-ray astrophysics and for his interest in the wider applications of IACTs, which made this study possible.

Facility: VERITAS.

\section{REFERENCES}

Acharya, B. S., Actis, M., Aghajani, T., et al. 2013, APh, 43, 3

Aleksić, J., Ansoldi, S., Antonelli, L. A., et al. 2014, A\&A, 572, A121

Armada, A., Cortina, J., \& Martinez, M. 2005, in Neutrinos and Explosive Events in the Universe, ed. M. M. Shapiro, S. Todor, \& J. P. Wefel (NATO Science Ser. II, Vol. 209; Dordrecht: Springer), 307

Berk, A., Anderson, G. P., Acharya, P. K., et al. 2006, Proc. SPIE, 6233, $62331 \mathrm{~F}$

Bodman, E. H. L., \& Quillen, A. 2015, arXiv:1511.08821

Bondi, H. 1970, QJRAS, 11, 443

Borra, E. F. 2012, AJ, 144, 181

Borra, E. F. 2013, ApJ, 774, 142

Boyajian, T. S., LaCourse, D. M., Rappaport, S. A., et al. 2015, MNRAS, in press (arXiv:1509.03622)

Cook, A. F., Weekes, T. C., Williams, J. T., \& O’Mongain, E. 1980, MNRAS, 193, 645

Covault, C. E. 2001, Proc. SPIE, 4273, 161

Davies, J. M., \& Cotton, E. S. 1957, J. Solar Energy Sci. Eng, 1, 16

Eichler, D., \& Beskin, G. 2001, AsBio, 1, 489

Fischer, D. A., Schwamb, M. E., Schawinski, K., et al. 2012, MNRAS, 419, 2900

Hankins, T. H., Kern, J. S., Weatherall, J. C., \& Eilek, J. A. 2003, Natur, 422, 141

Hanna, D., McCann, A., McCutcheon, M., \& Nikkinen, L. 2010, NIMPA, 612, 278

Hanna, D. S., Ball, J., Covault, C. E., et al. 2009, AsBio, 9, 345

Harp, G. R., Richards, J., Shostak, S., et al. 2015, arXiv:1511.01606

Hillas, A. M. 1985, Proc. ICRC, 3, 445

Høg, E., Fabricius, C., Makarov, V. V., et al. 2000, A\&A, 355, L27

Holder, J. 2015, arXiv: 1510.05675

Holder, J., Ashworth, P., LeBohec, S., Rose, H. J., \& Weekes, T. C. 2005, Proc. ICRC (Pune), 5, 387

Holder, J., Atkins, R. W., Badran, H. M., et al. 2006, APh, 25, 391

Howard, A., Horowitz, P., Mead, C., et al. 2007, AcAau, 61, 78

Howard, A. W., Horowitz, P., Wilkinson, D. T., et al. 2004, ApJ, 613, 1270

Ivezic, Z., Tyson, J. A., Abel, B., et al. 2008, arXiv:0805.2366

Lacki, B. C. 2014, MNRAS, 445, 1858

Lorimer, D. R., Bailes, M., McLaughlin, M. A., Narkevic, D. J., \& Crawford, F. 2007, Sci, 318, 777

Nagai, T., McKay, R., Sleege, G., \& Petry, D. 2008, Proc. ICRC, 3, 1437

Schaefer, B. E. 2016, ApJL, submitted (arXiv:1601.03256)

Schaefer, B. E., Vanderspek, R., Bradt, H. V., \& Ricker, G. R. 1984, ApJ, 283, 887

Schlafly, E. F., \& Finkbeiner, D. P. 2011, ApJ, 737, 103

Schmidt, B. 2012, in Proc. IAU Symp. 285, New Horizons in Time-Domain Astronomy (Cambridge: Cambridge Univ. Press), 9

Schuetz, M., Vakoch, D. A., Shostak, S., \& Richards, J. 2015, ApJL, submitted (arXiv:1512.02388)

Schwartz, R., \& Townes, C. 1961, Natur, 190, 205

Stone, R. P. S., Wright, S. A., Drake, F., et al. 2005, AsBio, 5, 604

Tellis, N. K., \& Marcy, G. W. 2015, PASP, 127, 540

Weekes, T. C., Badran, H., Biller, S. D., et al. 2002, APh, 17, 221

Wright, J. T., Cartier, K. M. S., Zhao, M., Jontof-Hutter, D., \& Ford, E. B. 2016, ApJ, 816, 17

Wright, S. A., Werthimer, D., Treffers, R. R., et al. 2014, Proc. SPIE, 9147 , 91470J 